\title{
Silencing ADAM10 inhibits the in vitro and in vivo growth of hepatocellular carcinoma cancer cells
}

\author{
SONGYANG LIU, WEI ZHANG, KAI LIU, BAI JI and GUANGYI WANG \\ Department of Hepatopancreatobiliary Surgery, The First Hospital, Jilin University, Changchun, Jilin 130021, P.R. China
}

Received December 31, 2013; Accepted September 4, 2014

DOI: $10.3892 / \mathrm{mmr} .2014 .2652$

\begin{abstract}
A disintegrin and metalloprotease 10 (ADAM10) is a transmembrane protein associated with metastasis in a number of types of cancer. Little is known, however, regarding the role of ADAM10 in hepatocellular carcinoma (HCC). The aim of the present study was to evaluate whether downregulation of ADAM10 effects HCC cell proliferation, cell cycle, cell migration and cell invasion. A recombinant small hairpin RNA expression vector carrying ADAM10 was constructed and then transfected into the HepG2 human HCC cell line. In vitro cell proliferation, cell cycle, cell migration and cell invasion, and in vivo tumor growth were determined following the downregulation of ADAM10 by RNA interference. The results revealed that downregulation of ADAM10 expression in HepG2 tumor cells using the RNA silencing approach significantly suppressed cell proliferation, cell migration and cell invasion in vitro, and tumor growth in vivo. Furthermore, ADAM10 silencing was able to significantly reduce constitutive phosphorylation of phosphoinositide 3-kinase (PI3K) and Akt, which implies that ADAM10 is, at least partially, involved in the activation of the PI3K/Akt signaling pathway. These results suggest that ADAM10 is important in regulating the proliferation and metastasis of HCC. Thus, ADAM10 is a promising therapeutic target for the prevention of tumor metastases in HCC.
\end{abstract}

\section{Introduction}

Hepatocellular carcinoma (HCC) is a leading cause of cancer mortality worldwide, with $>600,000$ novel cases diagnosed annually (1). Although there have been developments in surgical strategies and in clinical care, the overall outcome for patients with $\mathrm{HCC}$ remains poor, as $\mathrm{HCC}$ is commonly detected at a late stage, which precludes potentially curative

Correspondence to: Professor Guangyi Wang, Department of Hepatopancreatobiliary Surgery, The First Hospital, Jilin University, 71 Xinmin Street, Changchun, Jilin 130021, P.R. China

E-mail: wangguangyi1980@sina.com

Key words: hepatocellular carcinoma cancer, A disintegrin and metalloprotease 10, RNA silencing, tumor growth treatment (2). Thus, understanding the molecular mechanisms underlying the pathogenesis of this type of cancer, and developing sensitive and specific molecular markers and novel therapies is required.

A disintegrin and metalloproteases (ADAMs) are a family of zinc-dependent transmembrane proteins (3). The family encodes proteins that mediate cellular responses to environmental stress by interacting with various cell surface proteins and regulating diverse cellular processes. These proteins are involved in a range of human disease processes, such as cancer metastasis, inflammation and asthma (4,5). ADAM10, an important member of the ADAM family, is frequently upregulated in various types of cancer, including HCC, and is involved in cancer progression and metastasis (6-12). ADAM10 has been reported to exert an important role in cell migration, tumor development and metastasis by proteolytic shedding of cell surface proteins $(13,14)$. Recently, studies have revealed that RNA interference (RNAi)-mediated downregulation of endogenous ADAM10 inhibits adenoid cystic carcinoma cell growth and metastasis (15). In addition, Yang et al (10) demonstrated that ADAM10 is crucial in mediating the chemoresistance of HCC cells to doxorubicin. However, relatively little is known regarding the role of ADAM10 in HCC cells. Thus, in the present study, the feasibility of lentiviral vector-delivered small hairpin RNA (shRNA) against ADAM10 in the treatment of HCC was assessed in vitro and in vivo, and the molecular pathways involved were examined.

\section{Materials and methods}

Cell culture. HepG2 human HCC cell lines were purchased from the Type Culture Collection of Chinese Academy of Sciences (Shanghai, China) and were cultured in Dulbecco's modified Eagle's medium (DMEM) supplemented with $10 \%$ fetal bovine serum (FBS; Invitrogen Life Technologies, Carlsbad, CA, USA) in a humidified incubator in $5 \% \mathrm{CO}_{2}$ at $37^{\circ} \mathrm{C}$.

Preparation of plasmid-based ADAM10 shRNA vector and transfection of HepG 2 cells. An ADAM10 small interfering RNA (siRNA) sequence (CAGUGUGCAUUCAAGUCAA) and a scrambled control (NC) sequence that does not target any gene product and has no significant sequence similarity to human gene sequences (AATTCTCCGAACGTGTCACGT) 
were designed using the siRNA Target Designer software (version 2.0, Promega Corporation, Madison, WI, USA). Preparation of the RNAi vector expressing the human ADAM10 and NC shRNA was performed using the pSuper siRNA expression plasmid with the U6 promoter (Oligoengine, Seattle, WA, USA) according to previously described methods (16).

The HepG2 cells were transduced with either the specific ADAM10 shRNA vector or an empty plasmid using Lipofectamine $^{\mathrm{TM}}$ (Invitrogen Life Technologies) 2000 transfection reagent. $\mathrm{G} 418(300 \mu \mathrm{g} / \mathrm{ml})$ was employed to screen for stably transfected clones. ADAM10 expression levels were examined using reverse transcription-quantitative polymerase chain reaction (RT-qPCR) and western blot analysis using mouse anti human ADAM10 monoclonal antibodies to verify the silencing efficiency of the target gene following RNAi treatment. Cells with stable transfection and effective inhibition of the ADAM10 gene were termed ADAM10-RNAi cells, and cells with stable transfection of the NC sequence were termed NC-RNAi cells.

$R T-q P C R$. RT-qPCR analysis of ADAM10 transcripts in HepG 2 cells was conducted using TRIzol reagent (Invitrogen Life Technologies) according to the manufacturer's instructions. RNA was reverse-transcribed into cDNA using a Primescript ${ }^{\mathrm{TM}}$ RT reagent kit according to the manufacturer's instructions (Takara, Dalian, China). RT-qPCR was conducted using the SYBR Green fluorescent dye method and a Rotor Gene 3000 real-time PCR apparatus (Applied Biosystems Life Technologies, Foster City, CA, USA). ADAM10 gene-specific amplification was confirmed by PCR with specific primers (5'-CTGCCCAGCATCTGACCCTAA-3' and 5'-TTGCCATCAGAACTGGCACAC-3') and subjected to melting curve analysis. GAPDH served as an internal control for standardization. The primer sequences for $\beta$-actin were as follows: Forward: 5'-GATCATTGCTCCTCCTGAGC-3' and reverse: 5'-ACTCCTGCTTGCTGATCCAC-3'. The PCR conditions were as follows: Pre-denaturation at $95^{\circ} \mathrm{C}$ for $2 \mathrm{~min}$, followed by 40 cycles of denaturation at $95^{\circ} \mathrm{C}$ for $10 \mathrm{sec}$ and annealing/extension at $55^{\circ} \mathrm{C}$ for $20 \mathrm{sec}$. All RT-qPCR experiments were conducted in triplicate and were performed at $72 \mathrm{~h}$ after transfection. The data were analyzed using the comparative $\mathrm{Ct}$ method.

Western blot analysis. The cells were lysed by incubation on ice for $30 \mathrm{~min}$ in lysis buffer containing $25 \mathrm{mM}$ Tris- $\mathrm{HCl}(\mathrm{pH}$ 8.0), $1 \%$ Nonidet $\mathrm{P} 40,0.5 \%$ sodium deoxycholate, $0.1 \%$ sodium dodecylsulfate (SDS), $125 \mathrm{mM} \mathrm{NaCl}$ and Complete Protease Inhibitor Cocktail (Roche Diagnostics GmbH, Mannheim, Germany). Equal quantities of protein (15 $\mu \mathrm{g} /$ lane) from the cell lysates were separated on an 8-15\% SDS-polyacrylamide gel and transferred to nitrocellulose membranes (Santa Cruz Biotechnology, Inc., Santa Cruz, CA, USA). The membranes were incubated for $2 \mathrm{~h}$ in phosphate-buffered saline (PBS) with $0.1 \%$ Tween-20 and 5\% non-fat milk to block nonspecific binding. Subsequently, membranes were incubated with a mouse anti-ADAM10 monoclonal antibody (1:1,000; Santa Cruz Biotechnology, Inc., CA, USA), a mouse anti-Survivin monoclonal antibody (1:2,000; Santa Cruz, CA, USA), a mouse anti-Bcl-2 monoclonal antibody (1:1,500; Santa Cruz
Biotechnology, Inc.), a mouse anti-phosphorylated Akt (Ser473; p-Akt) monoclonal antibody (1:500; Cell Signaling Technology, Inc., Danvers, MA, USA), a mouse anti-Akt monoclonal antibody (1:2,000; Cell Signaling Technology, Inc.), a mouse anti-phosphorylated PI3K (Tyr458; p-PI3K) monoclonal antibody(1:1,000; Cell Signaling Technology, Inc.), a mouse anti-PI3K monoclonal antibody (1:3,000; Cell Signaling Technology, Inc.) and a mouse anti- $\beta$-actin monoclonal antibody (1:5,000; Cell Signaling Technology, Inc.) at room temperature for $2 \mathrm{~h}$. Following washing, anti-mouse secondary horseradish peroxidase-conjugated (1:10,000; Amersham Biosciences, Uppsala, Sweden) was added for $2 \mathrm{~h}$. Protein bands were visualized with an enhanced chimioluminescence reagent (ECL, Amersham, GE Healthcare, Velizy-Villacoublay, France). All assays using ADAM10 knockdown HepG2 cells were performed at $72 \mathrm{~h}$ after of transfection.

Cell proliferation assay. The 3-[4,5-dimethylthiazol2-yl]-2,5-diphenyltetrazolium bromide (MTT) colorimetric assay was used to screen for cell proliferation. Briefly, the cells were seeded in eight wells of 96-well plates at a density of $2 \times 10^{3}$ cells/well. Following culture for $24 \mathrm{~h}$, the cells were treated with ADAM10-RNAi or NC-RNAi. After $48 \mathrm{~h}$ culture, $20 \mu \mathrm{l}$ MTT $(5 \mathrm{mg} / \mathrm{ml})$ was added to each well followed by incubation at $37^{\circ} \mathrm{C}$ for $48 \mathrm{~h}$. Subsequently, centrifugation was performed at 2,000 x g for $10 \mathrm{~min}$. The supernatant was removed and $200 \mu \mathrm{l}$ dimethylsulfoxide was added to each well followed by agitation of the plates for $10 \mathrm{~min}$. The absorbance of each well was measured using a microplate reader (Molecular Devices Corp., Sunnyvale, CA, USA) at a wavelength of $490 \mathrm{~nm}$. The experiment was repeated three times. The mean proliferation of each treatment group is expressed as a percentage of the mean proliferation of the cells without any treatment.

Terminal deoxynucleotidyl transferase-mediated nick end labeling (TUNEL) assay. To measure the effect of RNAi-mediated ADAM10 downregulation on cell apoptosis, a TUNEL assay was conducted. Briefly, following HepG2 cell treatment with ADAM10 RNAi for $24 \mathrm{~h}$, cellular DNA fragmentation was measured with the ApoTag Red in situ Apoptosis Detection kit (Chemicon International, Temecula, CA, USA) according to the manufacturer's instructions. To quantify the apoptotic cells, TUNEL-positive cells were counted using a confocal microscope (BX2, Olympus Corporation, Tokyo, Japan). In addition, at the molecular level, the expression levels of Survivin and Bcl-2 apoptotic proteins were detected by western blotting as an additional indicator of apoptosis, as described above.

Wound-healing assay. To assess the effect of ADAM10 downregulation by RNAi on cell migration, a wound-healing assay was performed. A total of $1 \times 10^{5} \mathrm{HepG} 2$ cells were plated in 12-well plates in DMEM containing 10\% FBS. After $24 \mathrm{~h}$, a scratch was inflicted through the confluent cell monolayer, and then the cells were treated with ADAM10- RNAi or NC-RNAi respectively in $3 \mathrm{ml}$ complete medium. After $48 \mathrm{~h}$ treatment, the cells were stained with hematoxylin and eosin. The cells that invaded the wound line were observed with 
an inverted phase-contrast microscope (Leica DMR; Leica, Wetzlar, Germany). Triplicates were performed in all experiments.

Transwell invasion assay. Cell invasion was determined using Transwell chambers (BD Biosciences San Jose, CA, USA) consisting of polycarbonate membrane filters with a pore size of $8 \mu \mathrm{m}$. In brief, $2 \times 10^{5}$ HepG 2 cells in DMEM media with $0.5 \%$ FBS were added to the upper chamber containing $8 \mathrm{~mm}$ pore polycarbonate coated with $1 \mathrm{mg} / \mathrm{ml}$ Matrigel (BD Biosciences); the lower chamber was filled with media containing 5\% FBS. ADAM10-RNAi or NC-RNAi was added to the upper chambers, respectively. Cells without any drug treatment served as a control. After $16 \mathrm{~h}$ incubation, the upper membrane surface was scoured with a cotton-tipped swab. The invading cells on the lower membrane surface were fixed and stained with $0.5 \%$ crystal violet. Images of random fields (five per membrane) were captured using an inverted microscope (CKX31; Olympus Corporation) at magnification $\mathrm{x} 40$ for the calculation of the cell numbers. Furthermore, the cells were quantified by measuring the absorbance of dye extracts at $570 \mathrm{~nm}$ in $100 \mathrm{ml}$ Sorenson's solution containing $9 \mathrm{mg}$ tirsodium citrate, $195 \mathrm{ml} 0.1 \mathrm{M} \mathrm{HCl}, 305 \mathrm{ml}$ distilled water and $500 \mathrm{ml}$ of $90 \%$ ethanol. All experiments were performed in triplicate.

Tumor xenograft assay. All animal experiments were conducted according to the standards of animal care as outlined in the Guide for the Care and Use of Experimental Animals of Jilin University (Changchun, China), following a procedure approved by the Ethics Committees of the Disease Model Research Center at the First Hospital of Jilin University. Female BALBc mice, aged 4-5 weeks old were purchased from the Institute of Laboratory Animal Science of Jilin University (Changchun, China) and were maintained under specific pathogen-free conditions, and provided with food and water ad libitum. All animals were fed a normal pellet diet for one week prior to experimentation.

Exponentially growing HepG2 cells were harvested and a tumorigenic dose of $2 \times 10^{6}$ cells was injected intraperitoneally into the BALB mice. When tumors grew to an average volume of $75 \mathrm{~mm}^{3}$, the mice were randomly divided into ADAM10-RNAi, control (untreated) and NC-RNAi groups ( $n=8$ in each group). The control group received $1 \%$ polysorbate resuspended in deionized water. The other two groups were treated with either ADAM10-RNAi or NC-RNAi intraperitoneally injected on alternate days for three weeks. Tumor weight was measured when the mice were sacrificed by decapitation 21 days after treatment. Tumor volume was measured prior to the administration of the treatment injections and on days 7, 14 and 21 of treatment.

Statistical analysis. Statistical analysis between two samples was performed using Student's t-test. Statistical comparison of more than two groups was performed using one-way analysis of variance followed by a Tukey's post hoc test. All data are expressed as mean \pm standard deviation. SPSS ${ }^{\circledR} 19.0$ software (SPSS, Inc., Chicago, IL, USA) for Windows ${ }^{\circledR}$ was used for statistical analyses. $\mathrm{P}<0.05$ and $\mathrm{P}<0.01$ were considered to indicate a statistically significant difference.
A

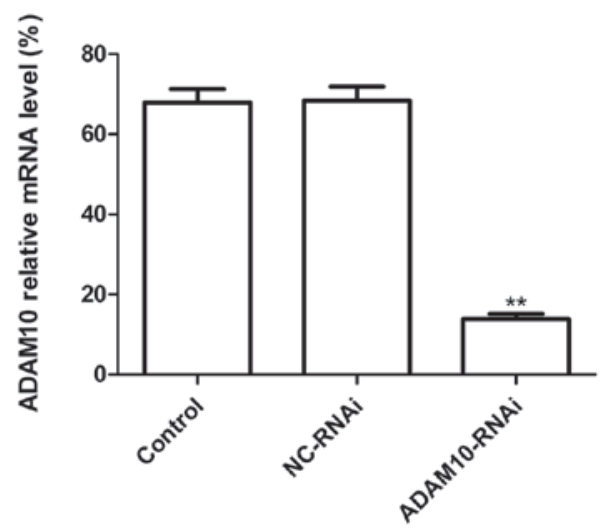

B

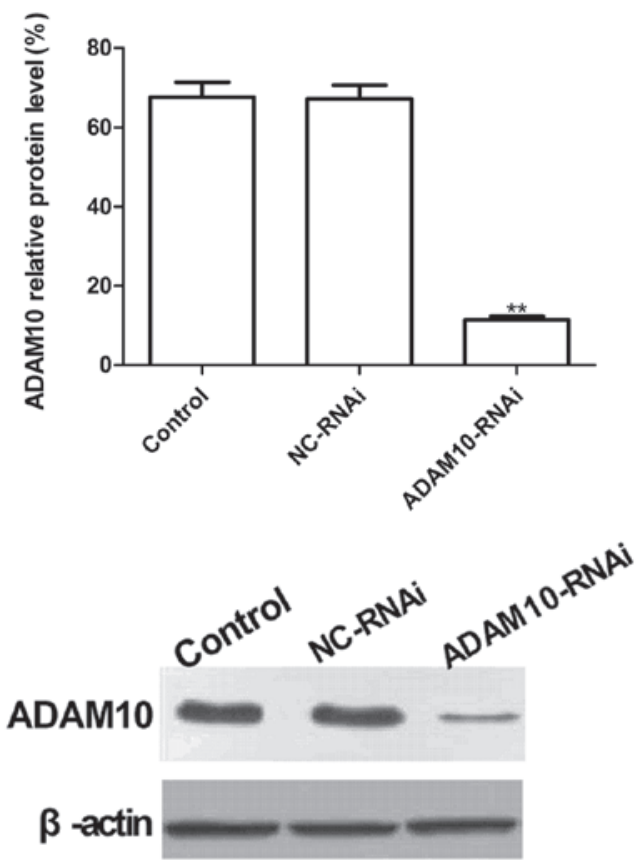

Figure 1. Suppression of ADAM10 expression in HepG2 hepatocellular carcinoma cells. (A) ADAM10 mRNA expression levels were determined by reverse transcription-quantitative polymerase chain reaction $48 \mathrm{~h}$ after ADAM10-RNAi silencing.(B) Western blot analysis of ADAM10 protein expression levels $48 \mathrm{~h}$ after RNAi silencing. ${ }^{* *} \mathrm{P}<0.01 \mathrm{vs.} \mathrm{control.} \mathrm{ADAM10,}$ A disintegrin and metalloprotease 10; RNAi, RNA interference; NC, scrambled control sequence.

\section{Results}

ADAM10-RNAi downregulates ADAM10 expression in HepG2 cells. To evaluate the silencing capacity of ADAM10, HepG2 cells were transfected with ADAM10-RNAi. RT-qPCR and western blotting were performed to detect the ADAM10 mRNA and protein expression levels at two days post-transfection. The results revealed no significant inhibition of ADAM10 expression in the NC-RNAi group, and no significant differences between the NC-RNAi and control (PBS) groups ( $\mathrm{P}>0.05)$. Conversely, ADAM10 mRNA and protein expression levels in the ADAM10RNAi group were significantly reduced following transfection as compared with the control group (Fig. 1A and $\mathrm{B}$; $\mathrm{P}<0.01$ ). These results demonstrate that ADAM10-RNAi significantly downregulated ADAM10 expression in HepG2 cells $(\mathrm{P}<0.01)$. 
A
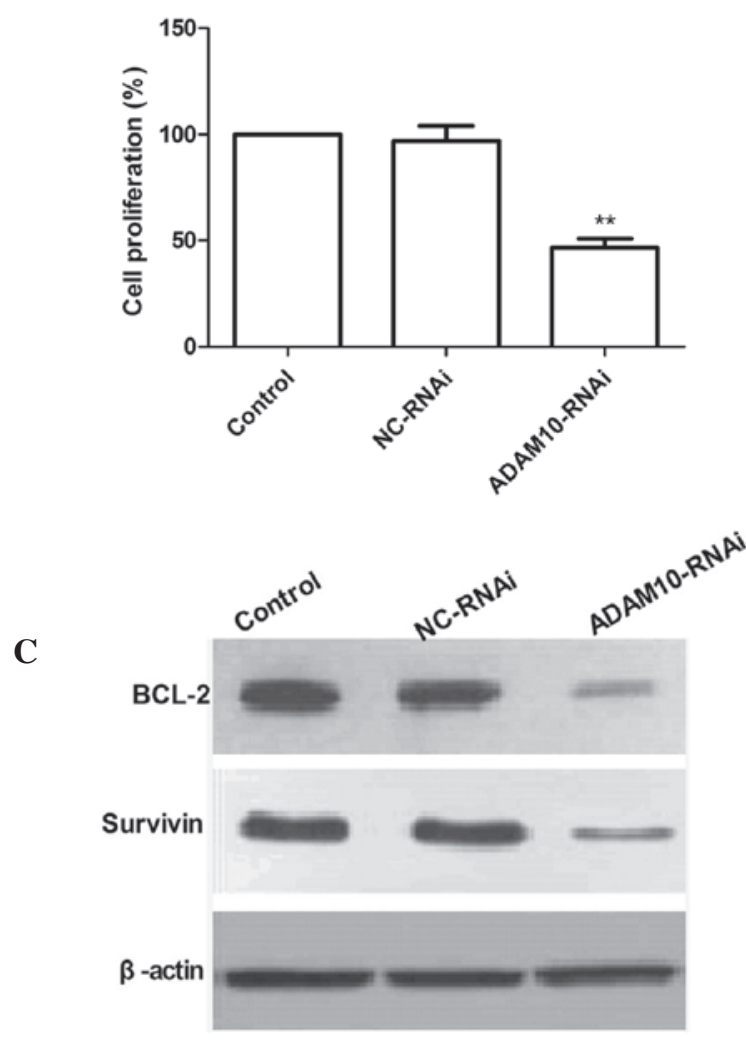

B
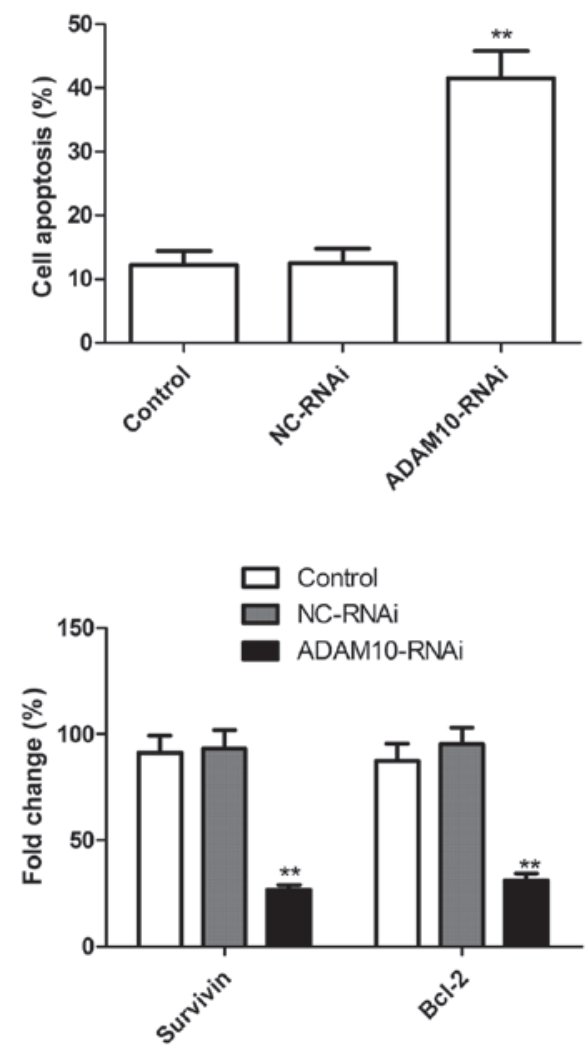

Figure 2. Silencing ADAM10 inhibited cell proliferation and cell apoptosis in vitro. Downregulation of ADAM10 by transfection with ADAM10-RNAi significantly (A) suppressed proliferation, (B) induced cell apoptosis and (C) affected apoptotic protein expression levels in HepG2 hepatocellular carcinoma cells. ${ }^{* *} \mathrm{P}<0.01$ vs. control. ADAM10, A disintegrin and metalloprotease 10; RNAi, RNA interference; NC, scrambled control sequence.

A

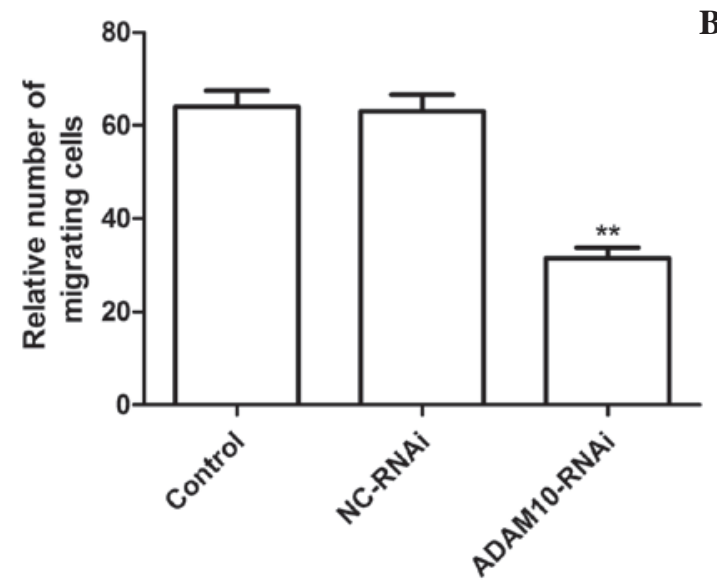

B

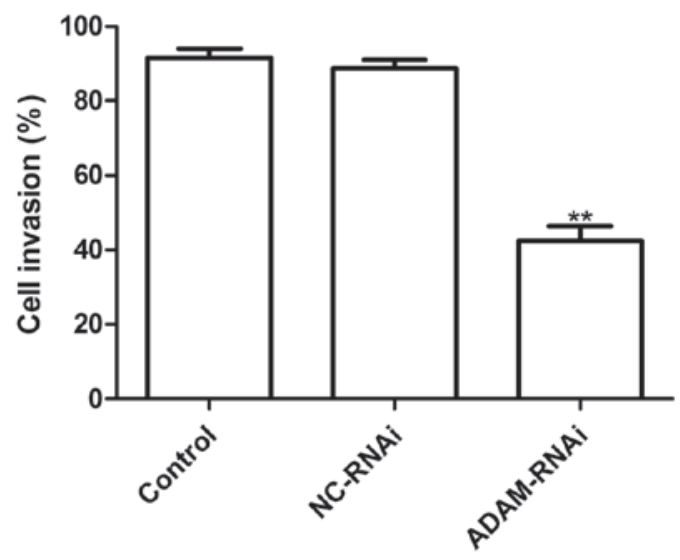

Figure 3. Silencing ADAM10 inhibited cell migration and cell invasion in vitro. Downregulation of ADAM10 by transfection with ADAM10-RNA interference inhibited (A) cell migration and (B) cell invasion in HepG2 hepatocellular carcinoma cells. ${ }^{* *} \mathrm{P}<0.01$ vs. control. ADAM10, A disintegrin and metalloprotease 10; RNAi, RNA interference; NC, scrambled control sequence.

Silencing ADAM10 reduces cell proliferation and induces cell apoptosis in Hep 62 cells. To examine whether the knockdown of ADAM10 expression exerts any effect on cell proliferation, an MTT assay was performed. As compared with the control and NC-RNAi cells, ADAM10-RNAi-transfected cells exhibited significantly reduced cell proliferation, which suggests the involvement of ADAM10 in HepG2 cell growth $(\mathrm{P}<0.02$; Fig. 2A).

In addition, the affect of ADAM10 gene silencing on HepG2 cell apoptotic ability was investigated by a TUNEL assay. ADAM10 silencing resulted in a significant increase in the number of apoptotic cells as compared with the control and 
A

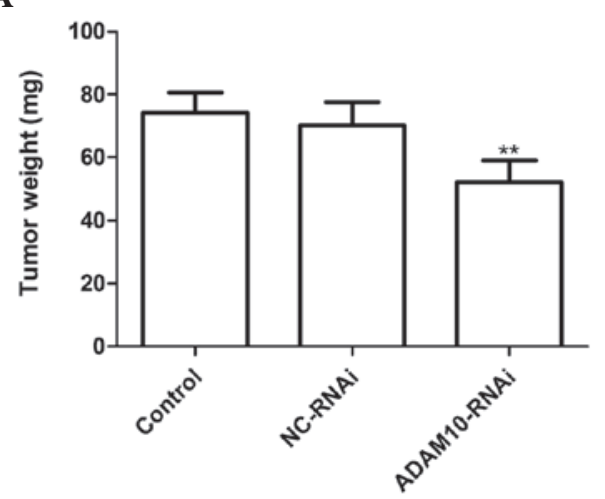

B

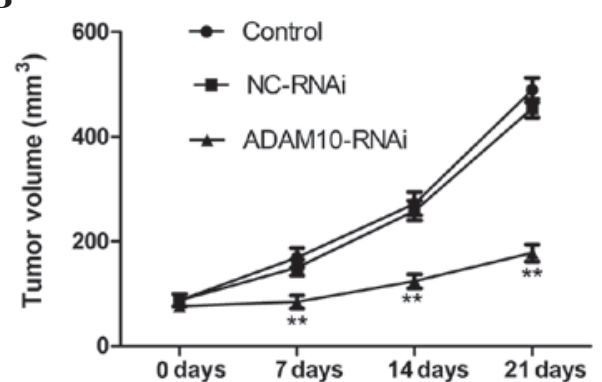

Figure 4. Silencing ADAM10 significantly suppressed HepG2 hepatocellular carcinoma cell tumor growth in vivo. (A) Tumor weight in xenograft mice 21 days after ADAM10 silencing. (B) Tumor volume curve. ${ }^{*} \mathrm{P}<0.05$ and ${ }^{* *} \mathrm{P}<0.01$ vs. control. ADAM10, A disintegrin and metalloprotease 10; RNAi, RNA interference; NC, scrambled control sequence.
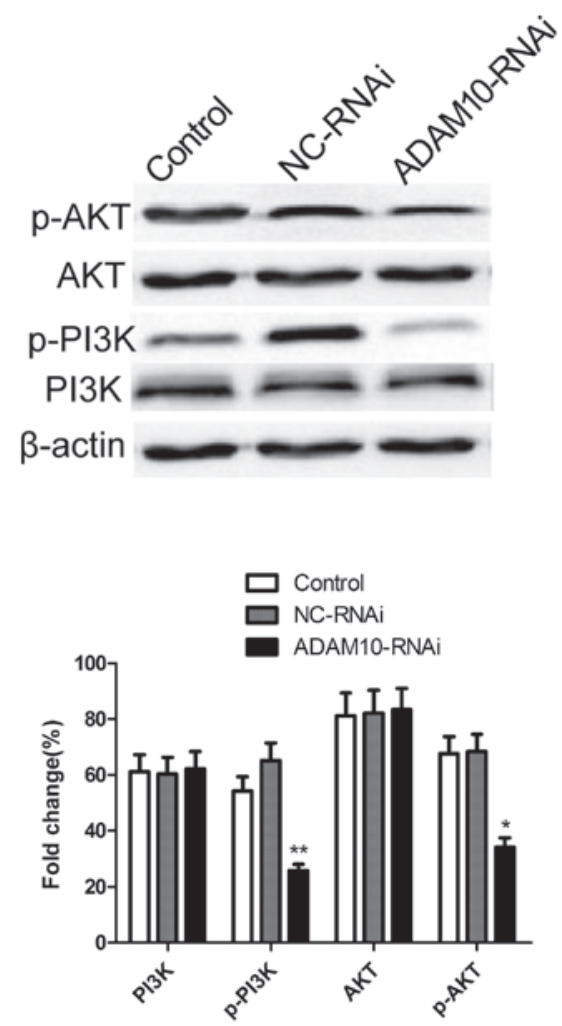

Figure 5. Silencing ADAM10 inhibited PI3K/Akt signaling in HepG2 hepatocellular carcinoma cells. The cells were treated with ADAM10-RNA interference for $24 \mathrm{~h}$. Western blot analysis was performed using specific antibodies against the indicated proteins. ${ }^{*} \mathrm{P}<0.05$ and ${ }^{*} \mathrm{P}<0.01$ compared with control. ADAM10, A disintegrin and metalloprotease 10; RNAi, RNA interference; NC, scrambled control sequence.
NC-RNAi groups (Fig. 2B; $\mathrm{P}<0.01$ ). No significant differences in the induction of $\mathrm{HCC}$ apoptosis were identified between the control and NC-RNAi groups.

To investigate the possible mechanism of the pro-apoptotic effect of silencing ADAM10 in HepG2 cells, Survivin and $\mathrm{Bcl}-2$ expression patterns were determined by western blotting. The results are shown in Fig. 2C. Silencing ADAM10 significantly reduced the expression levels of the Survivin and Bcl-2 apoptosis-inhibiting genes compared with control or NC-RNAi treatment. These data support the hypothesis that ADAM10 expression is required for promoting cell proliferation and inhibiting apoptosis'.

Silencing ADAM10 reduces cell migration and invasion in HepG2 cells. To ascertain the inhibitory effect of silencing ADAM10 on HepG2 cell migration, a wound-healing assay was performed. It was found that hepG2 cells transfected with ADAM10-RNAi migrated significantly less than those in the untreated cells or cells transfected with NC-RNAi (Fig. 3A P<0.01).

The ability of ADAM10 silencing to reduce the invasiveness of HepG2 cells was further investigated by a Transwell system assay. Invasion was found to be significantly reduced following ADAM10-RNAi treatment as compared with the control and NC-RNAi treatments ( $\mathrm{P}<0.01$; Fig. 3B).

Silencing ADAM10 suppresses tumor growth in vivo. The in vivo therapeutic efficacy of ADAM10 downregulation was assessed in female BALB mice bearing HepG2 tumors. The tumor weight in the ADAM10-RNAi group was significantly lower than that in the control and NC-RNAi groups $(\mathrm{P}<0.01$; Fig. 4A). In addition, tumor volume following treatment with ADAM10-RNAi was found to be significantly lower and increased at a markedly lower rate, as compared with the tumor volumes following control or NC-RNAi treatment $(\mathrm{P}<0.01$; Fig. 4B). These results indicate that downregulation of ADAM10 expression in HCC cells markedly suppressed tumor growth in mice.

Silencing ADAM10 inhibits the phosphorylation of Akt and PI3K in HepG2 cells. The antiapoptotic enzyme Akt was shown to be an important factor in apoptosis resistance in HCCs (17). Therefore, the effect of ADAM10 silencing on the phosphorylation of Akt and PI3K proteins was investigated by western blotting. As shown in Fig. 5, as compared with the control and NC-RNAi groups, silencing ADAM10 resulted in a significant suppression of Akt and PI3K phosphorylation. These results indicate that silencing ADAM10 induced apoptosis, to a certain extent, by suppression of the PI3K/Akt signaling pathway.

\section{Discussion}

ADAMs are involved in multiple cellular processes, such as cell proliferation, differentiation, migration and invasion $(4,5)$. Accumulating evidence suggests that ADAMs are also important in cell survival $(18,19)$. Certain members of the ADAM family, including ADAM9, ADAM10 and ADAM17, are closely involved in tumorigenesis, and the development and metastasis of tumors $(13,20,21)$. In addition, Yang et al (10) demonstrated that ADAM10 induces a prosurvival response in HCC cells 
upon doxorubicin treatment. Bai et al (22) found that expression of microRNA-122, as a negative regulator of ADAM10, sensitizes HCC cells to sorafenib, a multikinase inhibitor clinically effective against HCC. Yuan et al (23) observed that ADAM10 silencing resulted in inhibition of proliferation and migration as well as HepG2 human hepatoma cell invasion $(\mathrm{P}<0.05)$, a finding that is in concordance with the results of the present study. However, Yuan et al (23) did not investigate responses in an in vivo model. The results from the present study revealed that downregulation of ADAM10 expression using the RNA silencing approach in HepG2 tumor cells significantly suppressed cell proliferation, cell migration and cell invasion in vitro, and tumor growth in vivo. These results, combined with the findings from previous studies, suggest that ADAM10 may be a promising target for HCC anticancer therapy.

Although numerous studies have demonstrated that ADAM10 is involved in tumor cell proliferation and cell invasion, the underlying molecular mechanisms remain unclear. Extensive investigation has revealed that ADAM10 cleaves amyloid precursor protein $(24,25)$, a protein associated with the growth of several types of cell $(26,27)$. ADAM10 has also been reported to activate Notch signaling by suppressing the ectodomain shedding of $\Delta-1$, which subsequently results in a marked inhibitory effect on tumor cell proliferation (28). Endres et al (13) observed that ADAM10 cleaves collagen type IV in the basement membrane, a process associated with tumor metastasis and tumor cell proliferation. Murai et al (14) revealed that the cleavage of CD44 catalyzed by ADAM10 contributed to the migration and invasion of glioblastoma tumor cells. Yang et al (10) demonstrated that ADAM10 exerts an important role in modulating the chemosensitivity of HCC cells, which involved the activation of the PI3K/Akt signaling pathway. In the present study, the results revealed that downregulation of ADAM10 expression using RNA silencing suppressed the phosphorylation of Akt and PI3K, which indicates that ADAM10 silencing inhibits tumor cell growth, to a certain extent, by suppressing the activation of the PI3K/Akt signaling pathway. These studies imply that different mechanisms may be involved in the antiproliferative effects of ADAM10 against tumor cells.

In conclusion, in the present study, the results demonstrated that downregulation of ADAM10 expression using RNA silencing significantly suppressed the proliferation, cell migration and cell invasion in vitro, and tumor growth in vivo. Thus, considering the significance of cell invasion in metastatic progression, ADAM10 is a potential therapeutic target in the treatment of HCC.

\section{Acknowledgements}

This study was supported by the Jilin Provincial Science and Technology Research and Innovation Team Fund (grant no. JL2013011).

\section{References}

1. Parkin DM, Bray F, Ferlay J and Pisani P: Global cancer statistics, 2002. CA Cancer J Clin 55: 74-108, 2005.

2. Kern MA, Breuhahn K and Schirmacher P: Molecular pathogenesis of human hepatocellular carcinoma. Adv Cancer Res 86: 67-112, 2002.

3. Lu X, Lu D, Scully M and Kakkar V: ADAM proteins - therapeutic potential in cancer. Curr Cancer Drug Targets 8: 720-732, 2008.
4. Seals DF and Courtneidge SA: The ADAMs family of metalloproteases: multidomain proteins with multiple functions. Genes Dev 17: 7-30, 2003.

5. Klein $\mathrm{T}$ and Bischoff R: Active metalloproteases of the A Disintegrin and Metalloprotease (ADAM) family: biological function and structure. J Proteome Res 10: 17-33, 2011.

6. Kohga K, Takehara T, Tatsumi T, et al: Anticancer chemotherapy inhibits MHC class I-related chain a ectodomain shedding by downregulating ADAM10 expression in hepatocellular carcinoma. Cancer Res 69: 8050-8057, 2009.

7. Lee SB, Schramme A, Doberstein K, et al: ADAM10 is upregulated in melanoma metastasis compared with primary melanoma. J Invest Dermatol 130: 763-773, 2010.

8. Gaida MM, Haag N, Günther F, et al: Expression of A disintegrin and metalloprotease 10 in pancreatic carcinoma. Int J Mol Med 26: 281-288, 2010.

9. Wang YY, Ye ZY, Li L, et al: ADAM 10 is associated with gastric cancer progression and prognosis of patients. J Surg Oncol 103: 116-123, 2011.

10. Yang CL, Jiang FQ, Xu F and Jiang GX: ADAM10 overexpression confers resistance to doxorubicin-induced apoptosis in hepatocellular carcinoma. Tumour Biol 33: 1535-1541, 2012.

11. Ko SY, Lin SC, Wong YK, et al: Increase of disintergin metalloprotease 10 (ADAM10) expression in oral squamous cell carcinoma. Cancer Lett 245: 33-43, 2007.

12. Gavert N, Conacci-Sorrell M, Gast D, et al: L1, a novel target of beta-catenin signaling, transforms cells and is expressed at the invasive front of colon cancers. J Cell Biol 168: 633-642, 2005.

13. Endres K and Fahrenholz F: Upregulation of the alpha-secretase ADAM10 - risk or reason for hope? FEBS J 277: 1585-1596, 2010.

14. Murai T, Miyazaki Y, Nishinakamura H, et al: Engagement of CD44 promotes Rac activation and CD44 cleavage during tumor cell migration. J Biol Chem 279: 4541-4550, 2004.

15. Xu Q, Liu X, Chen W and Zhang Z: Inhibiting adenoid cystic carcinoma cells growth and metastasis by blocking the expression of ADAM 10 using RNA interference. J Transl Med 8: 136, 2010.

16. Brummelkamp TR, Bernards R and Agami R: A system for stable expression of short interfering RNAs in mammalian cells. Science 296: 550-553, 2002.

17. Leng J, Han C, Demetris AJ, Michalopoulos GK and Wu T: Cyclooxygenase-2 promotes hepatocellular carcinoma cell growth through Akt activation: Evidence for Akt inhibition in celecoxib-induced apoptosis. Hepatology 38: 756-768, 2003.

18. Kyula JN, Van Schaeybroeck S, Doherty J, et al: Chemotherapy-induced activation of ADAM-17: a novel mechanism of drug resistance in colorectal cancer. Clin Cancer Res 16: 3378-3389, 2010.

19. Rocks N, Estrella C, Paulissen G, et al: The metalloproteinase ADAM-12 regulates bronchial epithelial cell proliferation and apoptosis. Cell Prolif 41: 988-1001, 2008.

20. Wu K, Liao M, Liu B and Deng Z: ADAM-17 over-expression in gallbladder carcinoma correlates with poor prognosis of patients. Med Oncol 28: 475-480, 2011.

21. Zubel A, Flechtenmacher C, Edler L and Alonso A: Expression of ADAM9 in CIN3 lesions and squamous cell carcinomas of the cervix. Gynecol Oncol 114: 332-336, 2009.

22. Bai S, Nasser MW, Wang B, et al: MicroRNA-122 inhibits tumorigenic properties of hepatocellular carcinoma cells and sensitizes these cells to sorafenib. J Biol Chem 284: 32015-32027, 2009.

23. Yuan S, Lei S and Wu S: ADAM10 is overexpressed in human hepatocellular carcinoma and contributes to the proliferation, invasion and migration of HepG2 cells. Oncol Rep 30: 1715-1722, 2013.

24. Allinson TM, Parkin ET, Turner AJ and Hooper NM: ADAMs family members as amyloid precursor protein alpha-secretases. J Neurosci Res 74: 342-352, 2003.

25. Jorissen E, Prox J, Bernreuther C, et al: The disintegrin/metalloproteinase ADAM10 is essential for the establishment of the brain cortex. J Neurosci 30: 4833-4844, 2010.

26. Fan X, Liu Y, Jiang J, et al: miR-20a promotes proliferation and invasion by targeting APP in human ovarian cancer cells. Acta Biochim Biophys Sin (Shanghai) 42: 318-324, 2010.

27. Venkataramani V, Rossner C, Iffland L, et al: Histone deacetylase inhibitor valproic acid inhibits cancer cell proliferation via down-regulation of the alzheimer amyloid precursor protein. J Biol Chem 285: 10678-10689, 2010.

28. Zhao H, Zhu J, Cui K, et al: Bioluminescence imaging reveals inhibition of tumor cell proliferation by Alzheimer's amyloid beta protein. Cancer Cell Int 9: 15, 2009. 